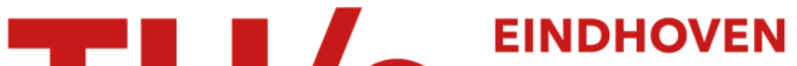 \\ UNIVERSITY OF \\ TECHNOLOGY
}

\section{Methods and approaches for RF circuit simulation and electromagnetic modelling}

\section{Citation for published version (APA):}

Kevenaar, T. A. M., Maten, ter, E. J. W., Janssen, H. H. J. M., \& Onneweer, S. P. (2002). Methods and approaches for $R F$ circuit simulation and electromagnetic modelling. (RANA : reports on applied and numerical analysis; Vol. 0222). Technische Universiteit Eindhoven.

\section{Document status and date:}

Published: 01/01/2002

\section{Document Version:}

Publisher's PDF, also known as Version of Record (includes final page, issue and volume numbers)

\section{Please check the document version of this publication:}

- A submitted manuscript is the version of the article upon submission and before peer-review. There can be important differences between the submitted version and the official published version of record. People interested in the research are advised to contact the author for the final version of the publication, or visit the $\mathrm{DOI}$ to the publisher's website.

- The final author version and the galley proof are versions of the publication after peer review.

- The final published version features the final layout of the paper including the volume, issue and page numbers.

Link to publication

\section{General rights}

Copyright and moral rights for the publications made accessible in the public portal are retained by the authors and/or other copyright owners and it is a condition of accessing publications that users recognise and abide by the legal requirements associated with these rights.

- Users may download and print one copy of any publication from the public portal for the purpose of private study or research.

- You may not further distribute the material or use it for any profit-making activity or commercial gain

- You may freely distribute the URL identifying the publication in the public portal.

If the publication is distributed under the terms of Article 25fa of the Dutch Copyright Act, indicated by the "Taverne" license above, please follow below link for the End User Agreement:

www.tue.nl/taverne

Take down policy

If you believe that this document breaches copyright please contact us at:

openaccess@tue.nl

providing details and we will investigate your claim. 


\title{
Methods and Approaches for RF Circuit Simulation and Electromagnetic Modelling
}

\author{
T.A.M. Kevenaar ${ }^{1}$, E.J.W. ter Maten ${ }^{2}$, H.H.J.M. Janssen ${ }^{1}$, S.P. Onneweer ${ }^{3}$ \\ 1 Philips Research Laboratories Eindhoven, Tom.Kevenaar@philips.com \\ 2 Philips Research Laboratories Eindhoven, Eindhoven University of Technology \\ ${ }^{3}$ Philips Semiconductors, Sunnyvale, CA, USA
}

\begin{abstract}
RF circuits and systems are gaining importance because we are moving further into a society where information is very important and should be available any time and anywhere. In this paper we give an overview of RF circuit simulation with an emphasis on noise simulation which is important functionality for RF designers. Due to the high frequency signals, the standard circuit formulation using Kirchhoff and lumped elements is not sufficient anymore to accurately predict the behaviour of a design and Maxwell's equations should be used. We give several approximations of Maxwell's equations and scenarios how the results can be incorporated in RF circuit simulation.
\end{abstract}

\section{Introduction}

High frequency applications are becoming increasingly important. This is caused by the fact that we are moving further into the information society where (digital) information is becoming very important. The first consequence is that large amounts of data should be transported, routed and processed at very high speeds. As an example, a switching array for optical data transmission may route 20 input signals each at $10 \mathrm{Gbit} / \mathrm{s}$ to one or many specified outputs. These switching arrays are still mainly implemented in silicon (or GaAs) and although we are dealing with digital circuits, the high frequency issues in this kind of circuits are analogue in nature and they have to be treated and analysed from an analogue viewpoint.

A second consequence of the increasing importance of information is the notion that this information should be available any time and anywhere. Therefore there is a tremendous increase in wireless networks that allow flexible access to a wide variety of information. The increase in functionality of, for example, cell phones leads to larger amounts of data to be exchanged. The same holds for the emerging in-home wireless digital networks.

Wireless transmission in general uses high frequency (RF) carriers usually in the range of 1-10 GHz. RF circuits are analogue circuits and should be treated as such.

Finally, many high frequency designs are aimed at a consumer market. This has severe consequences for the design process: where in the past there was time to build and measure several prototypes, nowadays the demands on 
time-to-market, time-to-quality, price, production volume and production yield, etc. are very severe. Furthermore, the increasing complexity and the decreasing size of these systems makes measuring extremely difficult and time consuming.

Therefore designers must be provided with analogue design environments that help them to design as quickly as possible a working circuit at first silicon. At the heart of these environments are the simulators. In order to predict the behaviour of a design as accurately as possible they should be provided with accurate models, not only of the non-linear components such as transistors but also of the physical structures which are part of the design.

In this paper we will give an overview of RF circuit simulation. First we will describe two main characteristics of RF circuits and RF designs. Next we will look in more detail at an approach to RF simulation that is becoming increasingly popular. This is followed by an explanation on why and how the physical implementation is incorporated in RF circuit simulation.

\section{Characteristics of RF circuits}

Clearly the most obvious characteristic of RF circuits is that they work at high frequencies. A consequence is that one has to use Maxwell's equations rather than Kirchhoff's equations and lumped models. Although clearly Kirchhoff's equations can be used without problems up to a certain frequencies, at RF frequencies they no longer accurately predict the behaviour of a design. The physical structures required to implement a design, such as tracks on an IC, connectors, etc., begin to play an important role in the total behaviour of the design. Since these structures in general do not have a regular shape, one has to resort to numerical methods to solve Maxwell's equations and somehow incorporate the results in a simulation.

A second characteristic is that RF signals have a broad but sparse spectrum [10] with a dynamic range of more than $60 \mathrm{~dB}\left(10^{6}\right)$. The weakest signals might be almost lost in noise while the strongest signals will introduce all kind of spurious intermodulation components due to non-linearities which are always present in a circuit. Noise and non-linear distortion translate to bit-error-rate in the transmitted data. Consequently, it is important that designers can predict the overall noise and distortion quickly and accurately.

\section{RF circuit simulation}

\subsection{Overview of RF building blocks and specifications}

Although RF systems can be quite complicated, they are typically built from a limited number of building blocks. When discussing RF simulation techniques it is important to first determine the special properties and characteristics of each building block and the information that should be obtained 
in a simulation. The most important blocks are mixers (which perform a frequency shift of the input signal, but also add non-linear intermodulation products and noise), amplifiers and filters (which distort the signal and add noise to it; power amplifiers may be strongly non-linear), dividers (strongly non-linear; modify a frequency reference signal), oscillators (generate a signal that serves as a frequency reference, often of very high accuracy; are autonomous circuits). In all cases non-linearity and noise are important issues. In the case of oscillators noise present in the circuit manifests itself as phase noise.

In the following section we will explain why and how these properties are specified.

\subsection{RF specifications}

In RF circuits the non-linearities are of course no different from non-linearities in other analog circuits. However they are usually expressed as intercept points like IP2 and IP3. The term 'intercept' originates from a graphical construction which can be used to determine IP2 and IP3. The numbers '2' and ' 3 ' refer to the order of the intermodulation product which they define. Clearly, these specifications should be determined using a full-nonlinear transient-like or harmonic balance-like simulation after which the result can be post-processed to yield the IP numbers.

More interesting is the noise in RF circuits. Noise consists of (usually) small unwanted signals in the circuit and originates in the devices that make up the circuit. For input/output circuits, a designer is usually interested in how much noise will be present or added in a certain, relatively narrow, frequency band of interest (FBOI). It will be the task of the simulator to accurately determine what the final noise spectrum in the FBOI looks like. A special thing in RF circuits is that spectra of input and noise signals are shifted around in the frequency band due to wanted (e.g. mixers) and unwanted non-linearities in combination with large signals. These signals can be internal to the circuit or be part of the input signal. Due to this noise folding, the noise in the FBOI might originate at completely different frequencies. The simulator must be able to handle noise folding.

Where in input/output circuits a designer is usually interested in the noise spectrum in a certain FBOI, when designing oscillators he is interested in the phase noise. As mentioned, an oscillator generates a frequency reference in the form of a periodic signal. Noise in the circuit causes the output signal to become noisy too. It is very important to notice that the noise might change the frequency of the output signal. This has severe consequences for simulation algorithms: noise is usually seen as a small perturbation on the noiseless solution which means that we can linearise the circuit. With oscillators this is no longer true as will be explained in the next Section. 


\subsection{Basic RF simulation methods}

Now that we have illustrated some important properties of RF circuits and signals it can be seen that conventional simulation methods like $\mathrm{AC}$ and transient simulation are not sufficient for simulating noise in RF circuits. AC simulation is fast but does not incorporate non-linearities nor frequency folding. Transient analysis could be used to simulate frequency folding but this would lead to prohibitively long simulation times if we want to obtain accurate information in the frequency domain. This is the reason why special RF simulation algorithms were developed.

The objective of RF circuit simulation is to obtain solutions for the network variables (the voltages, currents, charges and fluxes) in the circuit and to study the effects due to noise. All the newly developed RF simulation methods somehow use the Periodic Steady-State solution (PSS) as a starting point. Conceptually, the PSS solution can be seen as a generalisation of the DC solution: where the DC solution describes the voltages and currents after infinite time in a circuit containing only $D C$ sources, the PSS solution describes the voltages and currents after infinite time in a circuit containing only periodic sources (which include DC sources). As with the DC solution, the PSS solution is useful in its own right but it is also used as a basis for other analyses (for example, periodic AC and periodic noise). This presents a basic two-step approach:

- Firstly a (noiseless) PSS solution is determined which deals with the non-linearities in the circuit $[2,3,7,8,10,13,14,17-19]$,

- Secondly, a perturbation analysis is done around the PSS solution to analyse noise including frequency shifts $[1,4-6,16]$.

It is important to notice that the PSS algorithms make a distinction between forced and oscillatory problems. In the former the period of the solution is known beforehand while in the latter, determining the (exact) period is part of the problem. In general the oscillatory problems are more difficult to solve $[7,12,15]$. Also the solution of perturbed oscillatory problems may not be periodic at all.

The noiseless PSS problems are defined as finding a solution $\mathbf{x}(t)$ for systems of DAEs of the form

$$
\left\{\begin{array} { r l } 
{ \frac { d } { d t } \mathbf { q } ( t , \mathbf { x } ) + \mathbf { j } ( t , \mathbf { x } ) } & { = \mathbf { 0 } \in \mathbf { R } ^ { \mathbf { N } } } \\
{ \mathbf { x } ( 0 ) } & { = \mathbf { x } ( T ) }
\end{array} \text { or } \left\{\begin{array}{rl}
\frac{d}{d t} \mathbf{q}(\mathbf{x})+\mathbf{j}(\mathbf{x}) & =\mathbf{0} \in \mathbf{R}^{\mathbf{N}} \\
\mathbf{x}(0) & =\mathbf{x}(T)
\end{array}\right.\right.
$$

where $T>0$ is the period of the solution; $\mathbf{x}$ contains the node voltages and currents through voltage sources and through inductors; q describes the capacitor charges as well as fluxes through inductors; $\mathbf{j}$ describes currents, or voltages differences, as well as effects of sources. The explicit dependence on $t$, in the equations at the left, denotes that periodic sources are present in the circuit (forced problems) and therefore the period $T$ is known before hand. With oscillators (the autonomous equations at the right) this is not the case 
and the period $T$ should be solved together with $\mathbf{x}(t)$.

The PSS solution can be obtained in the frequency domain by determining its Fourier coefficients by a Harmonic Balance approach. This has the advantage that one can easily deal with circuit components that are characterised in the frequency domain which is not uncommon in RF applications. The time domain methods more easily deal with strong non-linearities in the circuit and have good convergence properties. Of these methods we mention Poincaré-map based methods, where increase in speed is obtained by applying vector acceleration methods such as Minimal Polynomial Extrapolation. Typical points of attention are: restarting at consistent solutions (i.e. satisfying the DAE-manifold), as well as dealing with multiple oscillation frequencies. Alternative methods are provided by (multiple) shooting methods, or by applying a finite difference method. All methods can be enhanced to deal with oscillatory systems in which $T$ is an additional unknown (and a gauge equation is added to the system).

The PSS-solution is useful to, for example, determine the non-linearity of the circuit such as IP2, IP3 etc. (see Section 3.2). For studying effects due to noise, the PSS solution is a first step in RF noise analysis in the two-step approach described before. An important effect of noise in RF circuits (see also Section 3.2) is noise folding: noise components are moved around the frequency band when they interact with other signals in the circuit. We now illustrate how a two-step approach based on a PSS solution is capable of reproducing this effect.

Assume that we determined the (forced) PSS solution $\mathbf{x}_{P S S}(t)$ for (1). To incorporate noise we add a perturbation term $\mathbf{n}(t)$ and get

$$
\frac{d}{d t} \mathbf{q}(t, \mathbf{x})+\mathbf{j}(t, \mathbf{x})+\mathbf{n}(t)=\mathbf{0} \in \mathbf{R}^{\mathbf{N}} .
$$

A natural approach would be to assume that a small $\mathbf{n}(t)$ also introduces a small deviation to the large signal solution $\mathbf{x}_{P S S}(t)$ and to linearise (2) by choosing $\mathbf{x}(t)=\mathbf{x}_{P S S}(t)+\mathbf{x}_{n}(t)$ and we find the following system for $\mathbf{x}_{n}(t)$ :

$$
\begin{aligned}
& \frac{d}{d t}\left(\mathbf{C}(t) \mathbf{x}_{n}\right)+\mathbf{G}(t) \mathbf{x}_{n}+\mathbf{n}(t)=\mathbf{0} \in \mathbf{R}^{\mathbf{N}} \\
& \mathbf{C}(t)=\left.\frac{\partial \mathbf{q}(t, \mathbf{x})}{\partial \mathbf{x}}\right|_{\mathbf{x}_{\mathrm{PSS}}}, \quad \mathbf{G}(t)=\left.\frac{\partial \mathbf{j}(t, \mathbf{x})}{\partial \mathbf{x}}\right|_{\mathrm{xPSS}} .
\end{aligned}
$$

It turns out that for forced systems this is a good approach because the period $T$ of the solution is completely determined by the input sources, and the homogenous problem only has the trivial solution. If we consider a typical Fourier component of the noise source, $\mathbf{n}(t)=\mathbf{U} e^{j \nu t}$, one may consider $\mathbf{y}_{n}(t)=e^{-j \nu t} \mathbf{x}_{n}(t)$ that satisfies a $T$-periodic system of equations

$$
\frac{d}{d t}\left(\mathbf{C}(t) \mathbf{y}_{n}\right)+[\mathbf{G}(t)+j \nu \mathbf{C}(t)] \mathbf{y}_{n}+\mathbf{U}=\mathbf{0} \in \mathbf{R}^{\mathbf{N}}
$$


(which is parametrized by $\nu$ ). It is clear that, for a single input frequency $\nu$, the solution $\mathbf{x}_{n}(t)$ contains frequencies of the form $\left(\nu+\omega_{k}\right)$ (in which $\omega_{k}=2 \pi k / T$ ), i.e. frequency folding occurs. If we allow for several input frequencies $\nu_{i}$, we can also say that a certain output frequency might originate from a large number of possible input frequencies. Hence, noise components at a certain frequency might end up in a different frequency band. This is why, for example, $1 / f$ noise which has its main energy at low frequencies, still plays an important role in RF circuits.

It is important to note that we described a linear perturbation analysis and we will not find contributions containing for example $\left(\nu_{1}+\nu_{2}+\omega_{k}\right),\left(\nu_{1}+\right.$ $\left.2 \nu_{2}+\omega_{k}\right)$ etc. This assumption is in general not a severe limitation when simulating noise in RF circuits.

When dealing with perturbed oscillatory systems

$$
\frac{d}{d t} \mathbf{q}(\mathbf{x})+\mathbf{j}(\mathbf{x})+\mathbf{n}(t)=\mathbf{0} \in \mathbf{R}^{\mathbf{N}}
$$

it is no longer possible to assume that small perturbations $\mathbf{n}(t)$ lead to small deviations in $\mathbf{x}_{P S S}(t)$ [An instructive example is provided by considering $y^{\prime}(t)+\cos (t) y(t)-1=0$, of which the inhomogeneous solution is not periodic at all; however, note that $y(t+2 \pi)$ still satisfies the differential equation]. The main reason is that the period of the large signal solution is influenced by $\mathbf{n}(t)$. This can lead to large (momentary) frequency deviations such that the difference between the noiseless and noisy solution can no longer be considered to be small.

In [5] a solution is given to deal with this problem by introducing an extra term which describes the frequency (or phase) shift of the solution due to $\mathbf{n}(t)$. Hence, rather than assuming $\mathbf{x}(t)=\mathbf{x}_{P S S}(t)+\mathbf{x}_{n}(t)$ as a solution for (6), we now assume $\mathbf{x}(t)=\mathbf{x}_{P S S}(t+\alpha(t))+\mathbf{x}_{n}(t)$, where $\alpha(t)$ is a non-trivial scalar function that has to be determined as part of the solution process and leads to the phase noise of the system. $\mathbf{x}_{n}$ represents the orbital deviation. In order to arrive at an expression for the phase- or time-shift function $\alpha(t)$ (assumed to be sufficiently smooth), we define $s=t+\alpha(t)$ and $\mathbf{y}(t) \equiv$ $\mathbf{x}_{\mathrm{PSS}}(s)=\mathbf{x}_{\mathrm{PSS}}(t+\alpha(t))$. We observe that $\mathbf{y}(t)$ satisfies

$$
\frac{\mathrm{d}}{\mathrm{d} t} \mathbf{q}(\mathbf{y})+\mathbf{j}(\mathbf{y})=\mathbf{C}(t+\alpha(t)) \mathbf{u}_{1}(t+\alpha(t)) \alpha^{\prime}(t),
$$

in which $\mathbf{u}_{1}(t)=\mathbf{x}_{\mathrm{PSS}}^{\prime}(t)$, being the tangent to the orbit. Clearly $\mathbf{y}(t)$ itself satisfies a perturbed differential equation. We note that $\mathbf{u}_{1}(t)$ satisfies the homogeneous part of (6), linearised around the noiseless PSS solution

$$
\frac{d}{d t}(\mathbf{C}(t) \mathbf{x})+\mathbf{G}(t) \mathbf{x}=\mathbf{0} \in \mathbf{R}^{\mathbf{N}},
$$

with $\mathbf{C}(t)$ and $\mathbf{G}(t)$ as defined in (4). It follows from Floquet theory $[5,11]$ that (8) has $N$ independent solutions (in which $\mathbf{u}_{1}$ coincides with our previously 
introduced one) $\mathbf{u}_{1}(t) e^{\mu_{1} t}, \ldots, \mathbf{u}_{m}(t) e^{\mu_{m} t}, \mathbf{u}_{m+1}(t), \ldots, \mathbf{u}_{N}(t)$. In case of a stable index 1 problem we can assume $\mu_{1}=0$ and $\operatorname{Re}\left(\mu_{i}\right)<0$ for $i \geq 2$. The adjoint system of (8)

$$
\mathbf{C}^{T}(t) \frac{d}{d t}(\mathbf{y})-\mathbf{G}^{T}(t) \mathbf{y}=\mathbf{0} .
$$

has similar properties: $\mathbf{v}_{1}(t) e^{-\mu_{1} t}, \ldots, \mathbf{v}_{m}(t) e^{-\mu_{m} t}, \mathbf{v}_{m+1}(t), \ldots, \mathbf{v}_{N}(t)$ are $N$ independent solutions. It should be noted that the vectors $\mathbf{v}_{i}(t)$ and $\mathbf{u}_{j}(t)$ satisfy a special bi-orthogonality relation with respect to $\mathbf{C}(t)$ and $\mathbf{G}(t)$, namely

$$
\mathbf{V}(t) \mathbf{C}(t) \mathbf{U}(t)=\left(\begin{array}{cc}
I_{m} & 0 \\
0 & 0
\end{array}\right), \quad \mathbf{V}(t) \mathbf{G}(t) \mathbf{U}(t)=\left(\begin{array}{cc}
J_{m}^{1} & 0 \\
J_{m}^{2} & J_{m}^{3}
\end{array}\right) .
$$

In applications, the noise (perturbation) term $\mathbf{n}(t)$ in (6) has the form $\mathbf{n}(t)=$ $B(\mathbf{x}(t)) \mathbf{b}(t)$. It seems natural to decompose $B\left(\mathbf{x}_{\mathrm{PSS}}(t+\alpha(t))\right) \mathbf{b}(t)$ into components along a basis of which one basic function is $\mathbf{C}(t+\alpha(t)) \mathbf{u}_{1}(t+\alpha(t))$ (see (7)). By multiplying (7) and $B(\mathbf{x}(t+\alpha(t))) \mathbf{b}(t)$ by $\mathbf{v}_{1}^{T}(t)$, the crucial bi-orthogonality implies a non-linear, scalar, differential equation for $\alpha(t)$

$$
\alpha^{\prime}(t)=-\mathbf{v}_{1}^{T}(t+\alpha(t)) B\left(\mathbf{x}_{\mathrm{PSS}}(t+\alpha(t))\right) \mathbf{b}(t), \quad \alpha(0)=0
$$

from which $\alpha(t)$ can be determined [The same bi-orthogonality also provides an elegant way to determine $\mathbf{v}_{1}(t)$, once $\mathbf{u}_{1}(t)$ is known]. Note that if $\mathbf{b}(t)=0$, for $t \geq t_{0}$, then $\alpha$ becomes a constant phase shift, and the phase shifted function $\mathbf{y}(t)$ solves (6) exactly for $t \geq t_{0}$. In general, even for small $\mathbf{b}$, the phase shift function $\alpha(t)$ may increase with time. Because (11) is non-linear, phase shifts from individual sources do not add up to give a group phase shift.

In the above we assumed deterministic disturbances prescribed by the time function $\mathbf{b}(t)$. In noise analysis, however, the noise is usually not described by time functions but by statistical properties such as mean and standard deviation. In [5,6] the (stationary) autocorrelation of $\mathbf{y}(t)$ is studied more closely (here the $*$ denotes complex conjugation). One derives (assuming real $\alpha(t)$ and $\mathbf{X}_{j}$ being the $j$-th Fourier coefficient of $\left.\mathbf{x}_{\mathrm{PSS}}(t)\right)$

$$
R_{\mathbf{y}}(\tau) \equiv \lim _{t \rightarrow \infty} E\left[\mathbf{y}(t) \mathbf{y}^{*}(t+\tau)\right]=\sum_{j=-\infty}^{\infty} \mathbf{X}_{j} \mathbf{X}_{j}^{*} e^{-i \omega_{j} \tau} R_{j}(\tau)
$$

with a corresponding relation between the spectral densities

$$
S_{\tilde{\mathbf{x}}_{\mathrm{PSS}}}(\omega)=\sum_{j=-\infty}^{\infty} \mathbf{X}_{j} \mathbf{X}_{j}^{*} S_{j}\left(\omega+\omega_{j}\right), \text { where } \int_{-\infty}^{\infty} \mathbf{X}_{j} \mathbf{X}_{j}^{*} S_{j}\left(2 \pi f+\omega_{j}\right) \mathrm{d} f=\mathbf{X}_{j} \mathbf{X}_{j}^{*}
$$

The interesting point is that the above formulas do not require the explicit evaluation of $\alpha(t)$ ! 'Only' the variance $\sigma^{2}(\tau)$ of $\alpha(t)$ is met, which can be 
related to the $\sigma^{2}$ of the individual source. This allows for deriving approximating expressions for $S_{j}(\omega)$, and also gives way to summing efficiently for getting group contributions.

In this section we summarised RF noise algorithms based on a two-step approach: a PSS analysis followed by a linear (for forced systems) or non-linear (oscillatory systems) perturbation analysis. Apart from accurate simulation algorithms, a circuit simulator needs accurate models. On the one hand there are the models of the non-linear components such as bipolar transistors, MOS transistors, etc. Although these models are very important they are outside the scope of this paper. On the other hand, for accurate RF simulation we also require models of physical structures which are somehow generated by solving Maxwell's equations. This will be the topic of the following section.

\section{Electromagnetic modelling for circuit simulation}

\subsection{Concept of lumped elements}

In the previous sections we described circuit analysis that is based on Kirchhoff's voltage and current law or KVL and KCL, respectively. However, Maxwell's equations are more fundamental and the electromagnetic (EM) field is the foundation of circuit theory and electronic modelling and simulation. In practice, a complete electromagnetic model of an electronic circuit is expensive to create and analyse and fortunately, electronic circuit theory has shown how to approximate many practical circuits by lumped element models: the energy-storage elements, (inductors and capacitors), and the dissipative elements (resistors) are connected to each-other and to sources or active elements within the circuit by conducting paths of negligible impedance. So apparently, the distributed effects, inherent to the solution of Maxwell's equations, in many real circuits can be represented by a few properly chosen lumped coupling elements. Circuit simulation is based on this concept of lumped elements, which ignores the electromagnetic interaction that is present within and between the physical circuit components and interconnections.

A first level of refinement to the lumped approach is to model the real physical interconnections (be it on-chip, in an IC package, a hybrid module or on a PCB) by means of lumped parasitic elements describing the conductor resistance and the capacitive and inductive coupling between the conductors. The ideal (lumped) circuit is extended with this parasitic network and can then be treated by the same network analysis and simulation tools. This approach can be used when the individual elements and the total circuit are small compared to the wavelength of the signals (quasi-static approach) [22]. For structures comparable in size to the wavelength there are two effects which will play a role which can not be taken into account using this approach: distributed effects (compared to lumped) and retardation effects from one part of the circuit to another. 
Firstly, we consider the distributed effects. In general the lumped representation of an element is valid if the region it occupies is small compared to the wavelength and when only one type of energy storage, either electric or magnetic is important in that region. If the electric energy storage in parts of a primarily inductive element, or magnetic energy in a primarily capacitive element, becomes important, the approach through classic circuit theory is to divide each element into sub-elements that can be treated as one or the other. A good example is the capacitive coupling between the turns of an inductor, which in a first approximation can be represented by adding a capacitive element across the terminals of the inductor. A further improvement is to add capacitive elements between each pair of adjacent turns.

Secondly, retardation effects, arising from the finite propagation time of electromagnetic effects across the circuit, can cause phase delays in the circuit. If there is an in-phase component of the induced electric field (due to changing magnetic fields) and magnetic field (due to the current), this represents an energy transfer, which is in fact the radiated energy. Another phenomenon that cannot easily be modelled with lumped parasitic elements is the presence of frequency dependent inhomogeneous current distributions in non-ideal conductors, e.g. due to skin effect.

Electromagnetic simulation is aimed at overcoming the limitations of the lumped element and lumped parasitics approach. Electromagnetic simulators build an accurate spatial model of the physical structures of the circuit. The spatial model is accompanied by the material properties of the structural elements: conductivity, permittivity and permeability. In order to incorporate EM effect in circuit simulation, ports (or pins) are attached to the physical structure, denoting the locations where e.g. lumped models of components or modules are to be attached. In many EM simulators, some approximation of Maxwell's equations is solved. Therefore, in the following section we will explain some often used approximations.

\subsection{Maxwell's equations and the Kirchhoff approximation}

Maxwell's equations describe the electromagnetic field and are given by:

$$
\begin{aligned}
\nabla \times \mathbf{E} & =-\frac{\partial \mathbf{B}}{\partial t}, \quad \text { (Faraday's law) } \\
\nabla \times \mathbf{H} & =\mathbf{J}+\frac{\partial \mathbf{D}}{\partial t}, \quad \text { (Ampère's law) } \\
\nabla \cdot \mathbf{B} & =0, \\
\nabla \cdot \mathbf{D} & =\rho, \quad(\text { Gauss' law) } \\
\mathbf{B} & =\mu \mathbf{H}, \quad \mathbf{D}=\epsilon \mathbf{E}, \quad \text { (constitutive relations) } \\
\mathbf{J} & =\sigma \mathbf{E}, \quad(\text { Ohm's law). }
\end{aligned}
$$

Here $\mathbf{E}$ and $\mathbf{H}$ are the electric and magnetic field, $\mathbf{B}$ and $\mathbf{D}$ are the magnetic and electric flux densities, $\mathbf{J}$ and $\rho$ are the current and charge density, 
and $\mu, \epsilon$ and $\sigma$ are the material parameters permeability, permittivity and conductivity, respectively. We can derive several approximations:

1. Assuming DC conditions $(\partial / \partial t=0)$ and taking the divergence of (13) we get $\nabla \cdot \mathbf{J}=0$, and with Gauss' theorem over a closed surface $S$

$$
\iiint_{V} \nabla \cdot \mathbf{J} d V=\oiiint_{S} \mathbf{J} \cdot d S=0 .
$$

Since the only current flowing out of the surface is in the wires, this gives Kirchhoff's current law (KCL), which simply states that the algebraic sum of currents flowing out of a circuit junction is zero.

From Faraday's law, we can introduce the potential (voltage) V according to $\mathbf{E}=-\nabla V$ and with Stoke's theorem over a closed loop $l$ we get:

$$
\iint_{A} \nabla \times \mathbf{E} \cdot d A=\oint_{l} \mathbf{E} \cdot d l=0 .
$$

This gives Kirchhoff's voltage law (KVL), which states that for any closed loop of a circuit, the algebraic sum of the voltages for the individual branches of the loop is zero. These two laws provide the basis for classical circuit theory.

2. If we only neglect the displacement current $\partial \mathbf{D} / \partial t$ in (13) we get the quasi-static approach. We still obtain the KCL by taking the divergence of (13). From (14) we can write $\mathbf{B}=\nabla \times \mathbf{A}$, where $\mathbf{A}$ is a magnetic vector potential. When we substitute this in (12), we get

$$
\nabla \times\left(\mathbf{E}+\frac{\partial \mathbf{A}}{\partial t}\right)=0=-\nabla \times \nabla V,
$$

which means that in this case we can also define a scalar electric potential $V$ by:

$$
\mathbf{E}=-\nabla V-\frac{\partial \mathbf{A}}{\partial t},
$$

where V fulfils the KVL. Taking the rotation of (12) and substituting (13) and taking the rotation of (13), we get the equations for $\mathbf{E}$ and $\mathbf{H}$ :

$$
\begin{aligned}
\Delta \mathbf{E} & =\mu \frac{\partial \mathbf{E}}{\partial t}=\mu \sigma \frac{\partial \mathbf{J}}{\partial t}, \\
\Delta \mathbf{H} & =-\nabla \times \mathbf{J}, \text { where } \Delta=\nabla^{2} .
\end{aligned}
$$

This gives the typical eddy current solutions with skin depth $\delta=1 / \sqrt{\pi \mu f \sigma}$ inside conductors with conductivity $\sigma$. Currents will run on the edge of the conductor within a depth $\delta$ and fields cannot penetrate any deeper in the conductor than this skin depth. This approach is required for frequencies $\omega>1 / \mu \sigma r^{2}$ where $r$ is the thickness of the interconnect. For 
frequencies high enough so that the current distribution is not uniform anymore (interconnect is thick compared to the skin depth), the resistance and internal reactance will become frequency dependent, since they will be determined by the skin depth. This approximation can also be seen as infinite wavelength approximation of the wave equation solution and is applicable when the wavelength is much larger than the dimension $d$ of the problem: $\lambda>d$ or frequencies $\omega<<c / d$, the so-called quasi-static approach. For higher frequencies, radiation losses become important and this is not taken into account by the quasi-static approach.

3. Clearly it is also possible to solve Maxwell's equations completely without any approximations which leads to the Helmholtz wave equations:

$$
\Delta \mathbf{E}-\frac{1}{c^{2}} \frac{\partial^{2} \mathbf{E}}{\partial t^{2}}=\mu \frac{\partial \mathbf{J}}{\partial t}+\nabla \frac{\rho}{\epsilon}, \Delta \mathbf{H}+\frac{1}{c^{2}} \frac{\partial^{2} \mathbf{H}}{\partial t^{2}}=-\nabla \times \mathbf{J}
$$

The solution of this set of equations is called the full-wave solution.

According to the analysis above, electromagnetic simulation tools may be characterised as either quasi-static or full-wave. Quasi-static simulators only consider free charges and currents on electrical conductors. By contrast, fullwave simulators account for the propagation of electromagnetic waves in free space and dielectric materials. Radiation losses are not naturally incorporated in the quasi-static model and radiated electromagnetic fields can only be approximated by post-processing on the calculated charges and currents. These phenomena are naturally incorporated in full-wave tools. A common problem in full-wave electromagnetic simulation tools is their computational complexity: because of the very fine spatial discretisation, the size of the mathematical equations that have to be solved is enormous. Reduced order modelling (ROM), the construction of a simplified system to approximate the original system with reasonable accuracy, will be necessary to cope with this [23-25]. Also for large quasi-static problems, ROM will be necessary.

\subsection{Electromagnetic simulation tools for RF circuits}

To assess the need for on-chip EM simulation let us assume that RF signals of interest for present-day businesses are in the frequency range of 1-10 GHz. Digital signals may have pulse edges in the order of $100 \mathrm{ps}$. For a straight TEM transmission-line consisting of ideal conductors embedded in a homogeneous dielectric medium the propagation speed of the signal is $c / \sqrt{\epsilon_{r}}$, where $\epsilon_{r} \approx 2-4$ for state of the art RFIC processes. As a result, the wavelength of the $10 \mathrm{GHz} \mathrm{RF}$ signal is of the order of $15-20 \mathrm{~mm}$. Likewise, the distance a digital pulse travels in $100 \mathrm{ps}$ is some $15-20 \mathrm{~mm}$. The die size is roughly $10 \mathrm{~mm}^{2}$. The maximum geometrical length of digital signal lines on the chip is comparable to the figures shown above. On the other hand, onchip RF interconnections are likely to be only a fraction of the die size and the largest components, spiral inductors, typically measure only $0.2-0.5 \mathrm{~mm}$ 
across. Consequently, from this perspective it is unlikely that it is necessary to apply full-wave electromagnetic simulation to on-chip components and interconnect structures. Long RF interconnections will be designed as coplanar wave-guides or similar structures and as long as the TEM propagation mode is dominant and the losses are small, the quasi-static approximation probably remains valid, even for very high frequencies.

Nevertheless, in order to tackle interconnect related problems Maxwell's equations (quasi-static and possibly full-wave) must be solved which can be done in the frequency domain or in the time domain. Within the frequency domain methods one assumes a harmonic time dependency, such that all time derivatives can be replaced by $j \omega$. Some of the most popular frequency domain methods are the Finite Difference (FDM) and Finite Elements (FEM) Method, as well as the Boundary Elements Method (BEM), Method of Moments (MoM) and Spectral Domain Analysis (SDA) [26,27]. The first two methods are differential equation schemes, the latter ones integral equation schemes. Well-known time domain methods are the Finite Difference Time Domain algorithm (FDTD) and the Time Domain Transmission Line Method (TDTLM).

The FDM approximates the differential operators by finite differences. It is easy to implement and applicable to general configurations, however has difficulty to handle curved boundaries. It may also need a large mesh volume to implement the absorbing boundary conditions (ABC) for unbounded problems.

In the FEM, the solution domain is discretized into elements [28]. Making use of interpolation functions (shape functions), each element is mapped into a basic standard element. The unknown fields are locally expressed in terms of the interpolation functions over each individual element. By applying a variational or Galerkin procedure, a set of algebraic equations, described by sparse matrices, is obtained. The Galerkin method is in fact a weighted residual procedure with trial functions equal to the weighting functions and is one of the most widely used methods. This method is also the most general. It can handle curved boundaries and arbitrary inhomogeneous material distributions. As with the FDM, one has to take care with $\mathrm{ABC}$ for unbounded problems.

When the FEM is applied to the boundary integral equations, this results in the BEM [29]. However, instead of large sparse matrices, where iterative solvers can be applied, dense matrices are obtained, which in general are smaller. This method can handle arbitrary curved boundaries and small mesh volumes, due to the integral equation approach. However, inhomogeneous material distributions are more difficult to handle.

The MoM also employs the method of weighted residuals [30]. The method starts by establishing a set of trial functions with one or more variables. The residuals are a measure of the difference between trial and true solution. The variable parameters are determined in a manner that guarantees a best fit of the trial functions based on a minimisation of residuals. 
In the SDA method, the integral equation is derived from the time-harmonic Maxwell's equations or the Helmholtz wave equations using Green's functions [31]. In this method only the surface of conductors needs to be discretized, resulting in a small dense matrix equation and thus a fast scheme. The major drawback is that it cannot easily be generalised, since Green's functions may not be available for a general configuration and inhomogeneous material distribution.

For integral equation methods, the so-called Fast Multi-pole Methods (FMM) can speed up the calculations and make large and complex geometries computable. The main idea behind these methods is the fact that the effect of all points working on all points yields a method with $O\left(N^{2}\right)$ computations, whereas if the points are well separated, one can cluster the "far-away" points. This yields a method of at most $O(N \log (N))$ [32].

The Finite-Difference Time-Domain (FDTD) method is currently one of the most popular approaches. As first proposed by Yee in 1966 [33], it uses the differential form of Maxwell's equations. Yee used an electric field (E) grid which was offset both spatially and temporally from a magnetic field (H) grid to obtain update equations that yield the present fields throughout the computational domain in terms of the past fields. The initial lack of attention, in spite of the simplicity and elegance of Yee's approach, can be attributed to the high computational cost. However, recently many shortcomings of the original FDTD method were alleviated leading to a near exponential growth in publications in the past ten years [34].

Finally, the TDTLM method models the spatial electromagnetic field in terms of a distributed transmission line network after discretizing the solution domain [35]. Basically this method has the same limitations as the FDTM method.

Unfortunately, however, most of the currently available 2.5D electromagnetic simulators (such as those based on MoM or FDTD) are only suited for relatively small interconnect structures due to large computing times.

\subsection{Coupling EM simulators to circuit simulators}

The purpose of our explanation on EM simulation was to include the influence of physical structures such as interconnect in RF circuit simulation. This means that somehow we have to connect the EM simulator, or the results thereof, to the circuit simulator. In this section we give some possible approaches.

A possible solution would be to tightly couple a circuit simulator with an EM simulator (co-simulation). The idea is based on an iterative approach in the time domain and requires costly computations and hence in general it is not an attractive approach. However, when antennas are integrated on silicon [36], electromagnetic and circuit analysis must be combined. By its very nature (conversion of electromagnetic radiation into electrical energy and visa versa) an antenna cannot be treated as a parasitic component and 
co-simulation will be necessary.

Another approach is to use an EM simulator as a stand-alone tool to generate a compact model which can be incorporated in the circuit simulator in addition to the lumped circuit components connected to the physical structure. This requires a robust method to catch the behaviour of the EM model at the ports. Most of the present EM simulators can produce Y- or S-parameter models that can be incorporated in a circuit simulator and they are most easily used in (linear or non-linear) frequency domain analyses such as AC and Harmonic Balance. For non-linear time-domain methods, such as PSS, we need to reflect the behaviour as a system of DAEs. There are a number of possible ways to couple this to the simulator. One can generate these equations directly from the S-parameters or via a behavioural modelling step. Some simulators calculate the impulse response of an S-parameter component by the inverse Laplace transform and apply numerical convolution during transient simulation.

Other tools are capable of creating an equivalent circuit model i.e. an RLC network. This type of model is more versatile but more difficult to generate. The size of the model could be a problem as well as its passivity (non-stable). Instead of mapping onto a real network, the model could also be described in terms of the G- and C matrices which can be incorporated in the circuit simulator.

Interesting work was done in coupling an FDTD simulator to the circuit simulator where a behavioural model approach was used to generate a lumped element model [38].

A limitation of using an EM simulator as stand-alone tool is that the actual EM model is never exercised in its circuit context, which means that the current distribution in the physical structures is not explicitly known. However, some EM simulators can reconstruct the geometrical current distribution and EM radiation from the currents at the model ports and create a visual rendition of them. To use this feature it is necessary to make the circuit simulation results available to the EM simulator [37].

\subsection{Future developments}

Currently the most common applications, where an EM simulator generates some kind of model for the circuit simulator, are the simulation of printed circuit boards and micro-wave circuits. The physical structures in these applications consist of planar metallisation structures on (almost) loss-less substrates. The most successful methods to analyze these usually large structures (e.g. complete multi-layer PCBs) are based on 2.5D boundary element analysis. These methods produce either a rational approximation of the Sparameters or a reduced lumped element model by using a fit procedure (Momentum-RF, Fasterix). In case of a lumped circuit model, the resulting circuit is in general not passive. Reduced order modelling techniques that guarantee stability, such as PVL [25], will need to be used. 
For modelling physical structures on an IC, one of the most promising solutions seems to be to adjust the tools and methods used for the planar structures. MoM and FDTD seem to be less favourable candidates for ICs, due to high computational cost. In order to be able to expand the above-mentioned tools for simulation of ICs, several problems need to be solved:

- 3D effects: due to the shape of IC interconnect, the effects of the side-walls have to be taken into account.

- Substrate effects: the actual IC interconnect behaviour can only be calculated when the effects of the silicon substrate are taken into account (displacement and induced (eddy) currents and concomitant losses). The silicon substrate cannot be approximated by an ideal conductive plane. The resistance of the substrate is high, leading to dispersion and frequency dependent attenuation in signal lines and to reduced q-factors in passive components, such as spiral inductors. A number of models to account for substrate effects have already been developed but need to be coupled to the interconnect analysis tools.

- These 3D and substrate effects increase the complexity of the problem. Therefore robust methods should be developed to reduce the complexity of the final model which is used in the circuit simulator. The stability of these models should be a point of attention.

\section{References}

1. W. Anzill, F.X. Kärtner, P. Russer: Simulation of the phase noise of oscillators in the frequency domain, AEÜ, Archive für Elektr. und Übertragungstechnik, Vol. 48-1, pp. 45-50, 1994.

2. H.G. Brachtendorf, G. Welsch, R. Laur, A. Bunse-Gerstner: Numerical steady state analysis of electronic circuits driven by multi-tone signals, Electrical Engineering 79, pp. 103-112, 1996.

3. H.G. Brachtendorf, R. Laur: Analyse des transienten Verhaltens von Oszillatoren durch eine inverse Charakteristikenmethode, ITG Workshop Mikroelektronik für die Informationstechnik, Darmstadt, Germany, 2000.

4. A. Demir, A. Sangiovanni-Vincentelli: Analysis and Simulation of noise in nonlinear electronic circuits and systems, Kluwer Academic Publ., Boston, USA, 1998.

5. A. Demir, A. Mehrotra, J. Roychowdhury: Phase noise in oscillators: a unifying theory and numerical methods for characterisation, DAC98, San Francisco, June 1998. Extended version IEEE Trans. on Circuits and Systems - I: Fund. Theory and Applics., Vol. 47-5, pp. 655-674, 2000.

6. A. Demir: Phase noise in oscillators: DAEs and coloured noise sources, Proc. ICCAD'98, Int. Conf. on Computer Aided Design, Nov. 8-12, 1998, San Jose, CA, USA, pp. 170-177, 1998.

7. S.H.M.J. Houben, E.J.W. ter Maten, J.M. Maubach, J.M.F. Peters: Novel time-domain methods for free-running oscillators, In: V. Porra, M. Valtonen, I. Hartimo, M. Ilmonen, O. Simula, T. Veijola: ECCTD'01 - Proceedings of the 15TH European Conference on Circuit Theory and Design, Helsinki University of Technology (ISBN 951-22-5571-5), Finland, pp III-393 - III-396, 2001. 
8. T.A.M. Kevenaar: Periodic Steady State Analysis using Shooting and Waveform-Newton, Int. J. Circuit Theory and Applics., Vol 22, pp. 51-60, 1994.

9. T.A.M. Kevenaar, E.J.W. ter Maten: RF IC Simulation: state-of-the-art and future trends, Proc. SISPAD'99 (ISBN 4-930813-98-0), Kyoto, Japan, pp. 7-11, 1999.

10. K. Kundert: Simulation methods for RF integrated circuits, Proc. ICCAD'97, San Jose, CA, USA, 1997.

11. R. Lamour: Floquet-Theory for differential-algebraic equations (DAE), ZAMM, Vol. 78-3, pp. S989-S990, 1998.

12. S. Lampe, H.G. Brachtendorf, E.J.W. ter Maten, S.P. Onneweer, R. Laur: Robust limit cycle calculations of oscillators, In: U. van Rienen, M. Günther, D. Hecht (Eds): Scientific computing in electrical engineering, Proc. SCEE2000, Warnemünde, Germany, Lecture Notes in Computational Science and Engineering 18, Springer Verlag, Berlin, Germany, pp. 233-240, 2001.

13. E.J.W. ter Maten: Numerical methods for frequency domain analysis of electronic circuits, Survey on Mathematics for Industry, Vol. 8, pp. 171-185, 1999.

14. K. Mayaram, D.C. Lee, S. Moinian, D. Rich, J. Roychowdhury: Overview of computer-aided analysis tools for RFIC: algorithms, features, and limitations, IEEE 1997 Custom Integrated Circuit Conference, Santa Clara, pp. 505-512, 1997.

15. R. Neubert, A. Schwarz: Efficient analysis of oscillatory circuits, In: U. van Rienen, M. Günther, D. Hecht (Eds): Scientific computing in electrical engineering, Proc. SCEE-2000, Warnemünde, Germany, Lecture Notes in Computational Science and Engineering 18, Springer Verlag, Berlin, Germany, pp. 225-232, 2001.

16. M. Okumura, H. Tanimoto, T. Itakura, T. Sugawara: Numerical noise analysis for nonlinear circuits with a periodic large signal excitation including cyclostationary noise sources, IEEE Trans. on Circuits and Systems - I: Fund. Theory and Applics., Vol.40-9, pp. 581-590, 1993

17. R. Pulch, M. Günther: A method of characteristics for solving multirate partial differential equations in radio frequency application, Preprint Nr. 00/07, IWRMM, Univ. Karlsruhe, subm. for publication, 2000.

18. M. Rösch, K. Antreich: Schnelle stationäre Simulation nichtlinearer Schaltungen im Frequenzbereich, AEÜ - Archive für Elektronik und Übertragungstechnik, 46, pp. 1-10 (1992).

19. J. Roychowdhury: Multi-time PDEs for dynamical system analysis, In: U. van Rienen, M. Günther, D. Hecht (Eds): Scientific computing in electrical engineering, Proc. SCEE-2000, Warnemünde, Germany, Lecture Notes in Computational Science and Engineering 18, Springer Verlag, Berlin, Germany, pp. 3-14, 2001.

20. D.A. Smith, W.F. Ford, and A. Sidi: Extrapolation methods for vector sequences, SIAM Review, 29(2), pp. 199-233, 1987.

21. R. Telichevesky, K. Kundert: Efficient $A C$ and noise analysis of two-tone $R F$ circuits, Proc. DAC'96, Las Vegas, 1996.

22. S. Ramo, J.R. Whinnery and T. Van Duzer, "Fields and Waves in Communication Electronics", Wiley, New York, 1996.

23. A. Odabasioglu and M. Celik, "PRIMA: Passive Reduced-Order Interconnect Macromodeling Algorithm", IEEE. Trans. Computer-Aided Design, Vol. 17, pp. 645-654, 1999. 
24. L. Knockaert and D. De Zutter, "Passive Reduced Order Multi-port Modeling: The Pade-Arnoldi-SVD Connection", Int. J. Electronics and Communications, no. 53, pp. 254-260, 1999.

25. P. Feldman and R.W. Freund, "Efficient Linear Circuit Analysis by Padé Approximation via the Lanczos Process", IEEE Trans. Computer-Aided Design, Vol. 14, pp. 639-649, May 1995.

26. G. Wang, "A Survey on Simulations of Metal-Insulator-Semiconductor Interconnects", Stanford internal report, Nov. 1998, http://wwwtcad.stanford.edu/ gaofeng/research/mis_review.htm

27. T.H. Hubing, "Survey of Numerical Electromagnetic Modeling Techniques", Univ. of Missouri-Rolla internal report, Sep. 1991, http://www.emclab.umr.edu/pdf/TR91-1-001.pdf

28. P.P. Silvester and R.L. Ferrari, "Finite Elements for Electrical Engineers", Cambridge Univ. Press, 1996.

29. C.A. Brebbia, J.C. Telles and L.C Wrobel, "Boundary Element Techniques", Berlin: Springer-Verlag, 1984.

30. R.F. Harrington, "Field Computation by Moment Methods", IEEE Press Series on EM Waves, 1993.

31. T.G. Livernois and P.B. Katehi, A Generalized Method for Deriving the Spacedomain Green's Function in a Shielded, Multi-layer Substrate Structure with Applications to MIS Transmission Lines", IEEE Trans. Microwave Theory Tech., Vol. MTT-37, pp. 1761-67, 1989.

32. W.C. Chew, J.M. Jin, E. Michielssen and J.M.Song (Editors), "Fast and Effcient Algorithms in Computational Electromagnetics", Artech House, Boston, MA, 2001.

33. K.S. Yee, "Numerical solution of initial boundary value problems involving Maxwell's equations in isotropic media", IEEE Trans. Antennas and Propagation, Vol. 14, pp. 302-307, 1966.

34. A. Taflove (Editor), "Advances in Computational Electrodynamics: The FiniteDifference Time-Domain Method", Artech House, Boston, MA, 1998.

35. P.B. Johns, "A Symmetrical Condensed Node for the TLM Method", IEEE Trans. Microwave Theory Tech., Vol. MTT-35, pp. 370-377, 1987.

36. D. Leenaerts, G. Gielen and R.A. Rutenbar, "CAD Solutions and Outstanding Challenges for Mixed-Signal and RF IC-Design", Proc. ICCAD 2001.

37. R. du Cloux, G.P.J.F.M. Maas, A.J.H. Wachters, R.F. Milsom and K.J. Scott, "Fasterix, an environment for PCB simulation", Proc. 11th Int. Conf. On EMC, Zürich, Switzerland, 1993.

38. P.B.L. Meijer, "Generalized Neural Networks for Modeling Non-linear Dynamic Multi-demensional Systems in Time and Frequency Domain", Philips Research Internal Report 6726, 1993. 